This item was submitted to Loughborough's Research Repository by the author.

Items in Figshare are protected by copyright, with all rights reserved, unless otherwise indicated.

\title{
Concurrent validity of ActiGraph-determined sedentary time against the activPAL under free-living conditions in a sample of bus drivers
}

PLEASE CITE THE PUBLISHED VERSION

http://dx.doi.org/10.1080/1091367X.2017.1335204

\section{PUBLISHER}

(C) Taylor \& Francis

\section{VERSION}

AM (Accepted Manuscript)

\section{PUBLISHER STATEMENT}

This work is made available according to the conditions of the Creative Commons Attribution-NonCommercialNoDerivatives 4.0 International (CC BY-NC-ND 4.0) licence. Full details of this licence are available at: https://creativecommons.org/licenses/by-nc-nd/4.0/

\section{LICENCE}

CC BY-NC-ND 4.0

\section{REPOSITORY RECORD}

Varela-Mato, Veronica, Thomas E. Yates, David J. Stensel, Stuart J.H. Biddle, and Stacy A. Clemes. 2019. "Concurrent Validity of Actigraph-determined Sedentary Time Against the Activpal Under Free-living Conditions in a Sample of Bus Drivers". figshare. https://hdl.handle.net/2134/25709. 
1 Concurrent validity of ActiGraph-determined sedentary time against the activPAL under 2 free-living conditions in a sample of bus drivers

3 Running head: SEDENTARY BEHAVIOUR MEASUREMENT

4

5

6

7

8

9

10

11

12

13

14

15

16

17

18 
Abstract

This study explored the validity of ActiGraph-determined sedentary time $(<50 \mathrm{cpm}$, $<100 \mathrm{cpm},<150 \mathrm{cpm},<200 \mathrm{cpm},<250 \mathrm{cpm}$ ) compared with the activPAL in a free-living sample of bus drivers. 28 participants were recruited between November 2013 and February 2014. Participants wore an activPAL3 and ActiGraph GT3X+ concurrently for 7 days and completed a daily diary. Time spent sedentary during waking hours on workdays, non-workdays, during working-hours and non-working hours were compared between instruments. During working hours, all ActiGraph cut-points significantly underestimated sedentary time $(p<0.05)$, whereas during non-working hours the $<50 \mathrm{cpm}$ cut-point demonstrated the closest agreement (ActiGraph sedentary time: $250 \pm 75$ minutes vs activPAL sedentary time: $236 \pm 65$ minutes). ROC analyses revealed that on workdays and non-workdays the ActiGraph cut-points exhibited relatively low sensitivity (all $<0.62$ ) and specificity (all $<0.49$ ) values. The use of the ActiGraph to measure sedentary time in this understudied, highly sedentary and at risk occupational group is not recommended.

Key words: Bus drivers, sedentary behaviour, validation, ActiGraph, activPAL. 


\section{Introduction}

Sedentary behaviour, described as any sitting or reclining posture with an energy expenditure $\leq 1.5$ MET's during waking hours (SBRN, 2012), has been linked to numerous adverse health outcomes, including obesity, type 2 diabetes, metabolic syndrome, some cancers and mortality from all causes and cardiovascular disease (Wilmot et al., 2012; Biswas et al., 2015). High levels (between $50-60 \%$ of waking hours) of sedentary time have been identified in office workers (Thorp et al., 2012; Brown et al., 2013; Clemes et al., 2015) and bus drivers (Wong et al., 2014; Varela-Mato et al., 2015), which put them at greater risk of co-morbidities and mortality in comparison to other occupational groups (John et al., 2006; Katzmarzyk et al., 2009; Dunstan et al., 2013).

Accelerometers have been used to overcome the limitations of self-report instruments and are commonly used in surveillance studies and interventions to quantify both physical activity and sedentary time. In addition, studies have shown a robust relationship between accelerometer-determined sedentary time and health outcomes (Healy et al., 2008; Kozey-Keadle et al., 2011). The use of accelerometry to provide an objective estimate of sedentary time, in addition to physical activity, has been widespread, with the ActiGraph being one of the most popular measurement tools within the literature (Kozey-Keadle et al., 2011; Hart et al., 2011; Berendensen et al., 2014). The ActiGraph is a small lightweight device that traditionally has been worn on an elastic belt on the hip. This device measures raw acceleration data by capturing the frequency and amplitude of the acceleration of the body segment to which it is attached (Atkin et al. 2012). Once the monitoring period is finished, these data can be clustered into sedentary, light, moderate and vigorous activities during the post-processing analysis (Atkin et al. 2012). However, 
ActiGraphs do not measure posture and instead sedentary time is estimated using a lack of movement counts. Several different thresholds (cut-points) have been applied to predominantly the vertical axis of the ActiGraph accelerometer, to estimate sedentary time (Kozey-keadle et al., 2011; Hart et al., 2011; Ridgers et al., 2012). A cut-point of less than 100 counts per minute (cpm) has been the most widely adopted to define sedentary time (Atkin et al., 2012) in studies ranging from clinical interventions to large scale epidemiological studies (Matthews et al., 2008; Healy et al., 2011). However, this cut-point was not empirically derived.

The activPAL is a lightweight device that is attached to the anterior aspect of the thigh. This contains a uni-axial accelerometer which responds to signals related to gravitational forces and provides information on thigh inclination (Kozey-Keadle et al., 2011). The activPAL has been shown to be a highly sensitive and valid measure of posture (Kozey-Keadle et al., 2011; Grant et al., 2006; Hart et al., 2011), overcoming the limitations of other accelerometers used to estimate sedentary time (e.g. the ActiGraph). However, literature studying the agreement between the ActiGraph and activPAL when measuring sitting time in a free-living environment is limited (Ridgers et al., 2012; Decker et al., 2013).

As sedentary behaviours are ubiquitous in most adults' day to day lives, accurately measuring sedentary time is important to establish dose-response relationships with health, to determine sedentary behaviour levels and patterns, and to identify behaviour domains to target in interventions. It is therefore important to assess the outputs from available instruments to measure sedentary time (ActiGraph and activPAL) taking into account different characteristics of each occupational group; as different job-related behaviours and working-environments might have an impact on the monitors' ability to correctly identify 
sedentary time. Most validation studies (comparing the ActiGraph against the activPAL) in adults have been conducted under controlled laboratory conditions, which have typically simulated computer-based working environments (Kozey-Keadle et al., 2011; Hart et al., 2011; Berendsen et al., 2014). No validation studies have been conducted in occupational drivers such as bus drivers, whereby the continuous vehicle accelerations and decelerations may have an impact on the accelerometers sensitivity to identify true sedentary time.

Indeed, of the limited research available examining bus drivers' sedentary time using accelerometry, vast differences in sedentary time have been reported across studies using different measurement tools. For example, using the ActiGraph accelerometer, Wong et al (2014) reported that bus drivers accumulated 8 hours/day of sedentary time on workdays (using a cut-point of $<150$ counts/minute $(\mathrm{cpm})$ ), whereas Varela-Mato et al (2016) reported that bus drivers accumulated 12 hours/day of sitting on workdays, using the activPAL. The largest differences in sedentary time observed between studies appeared to occur during working hours, with bus drivers reportedly spending up to 4 hours whilst at work sedentary in the study by Wong et al. (2014), whereas in the study by Varela-Mato et al. (2016), bus drivers spent approximately 7 hours sitting whilst at work. Whilst information on the drivers work pattern, in terms of hours spent driving, the number of breaks, and distance between stops is not available for a detailed comparison between studies, the large differences in sedentary times observed between these studies warrants further investigation into any potential differences between the measurement tools.

Despite the ActiGraph being used to assess sedentary time in bus drivers (Wong et al., 2014), no evidence currently exists on the validity of this measurement tool in this specific population. Due to the nature of their occupation which typically promotes 
prolonged periods of sitting, and their higher levels of disease risk (John et al., 2006; Joshi et

112 al., 2013; Wong et al., 2014) bus drivers are an important group to study in sedentary

113 behaviour research. This study therefore aims to explore the validity of ActiGraph-

114 determined sedentary time $(<50 \mathrm{cpm},<100 \mathrm{cpm},<150 \mathrm{cpm},<200 \mathrm{cpm},<250 \mathrm{cpm})$ in

115 comparison to the activPAL in a free-living sample of bus drivers. This study will further our

116 understanding of the validity of the ActiGraph as a sedentary behaviour measurement tool

117 in a different occupational group, considering their working environment.

\section{Methods}

\section{Participants}

A convenience sample of bus drivers was recruited from a bus company within the

East Midlands, UK, via leaflets and personal approaches by the researcher. Participants were recruited at their place of work during their breaks, with the manager's consent, between November 2013 and February 2014. Participants drove single-decker buses travelling local routes with an average of 20 stops per route. Participants breaks varied in number between

1261 and 4 depending on the route and their shift (average shift duration: 9 hours/day). Written consent was obtained from 35 eligible full-time bus drivers ( $42 \%$ of the driving workforce). Ethical approval was granted from the Loughborough University Ethical Advisory Committee. 

portable stadiometer (Seca 206, Oxford, UK). Body composition and weight were assessed using a Tanita BC-418 MA Segmental Body Composition Analyzer (Tanita, UK Ltd). BMI was calculated as $\mathrm{kg} / \mathrm{m}^{2}$.

\section{Measurement of Sedentary Behaviour}

The activPAL was initialised and downloaded using activPAL Professional v.7.2.29

software (device firmware version 3.107). It was attached directly to the skin on the midline of the anterior aspect of the right thigh using a hypoallergenic medical dressing (BSN Hypafix). The activPAL3 determines posture using information derived from accelerations of the thigh, including the gravitational component, using a triaxial accelerometer (Atkin et al., 2012). Due to its precision to differentiate between postures during free-living activities (Godfrey et al., 2007), the activPAL3 has been shown to be a valid measure of time spent sitting/lying, standing and walking in adults (Kozey-Keadle et al., 2011; Grant et al., 2006; Hart et al., 2011). This monitor has been used as the criterion measure in studies investigating the validity of waist-worn accelerometers for estimating sedentary time (Oliver et al., 2010; Ridgers et al., 2012). The activPAL3 was therefore used as the criterion measure in this study.

The ActiGraph GT3X+ was worn on an elasticated belt on the waist above the mid-

150 line of the right thigh. The device was initialised at a frequency of $100 \mathrm{HZ}$ and downloaded using ActiLife software v6.11.8 and firmware version 2.0.0. Several sedentary behaviour cutpoints ranging from 0 to $500 \mathrm{cpm}$, applied to the vertical axis, have been proposed in the literature (Atkin et al., 2012; Kozey-Keadle et al., 2011), with the $<100 \mathrm{cpm}$ cut-point being the most commonly used (Atkin et al., 2012; Healy et al., 2008; Brocklebank et al., 2015). In 
this study ActiGraph data were downloaded in 60-seconds epochs and sedentary time was defined as the sum of minutes where the monitor output (from the vertical axis) was $<50 \mathrm{cpm},<100 \mathrm{cpm},<150 \mathrm{cpm},<200 \mathrm{cpm},<250 \mathrm{cpm}$ and $<300 \mathrm{cpm}$.

Participants were advised to wear both devices concurrently and continuously over a seven-day period, except during water-based activities. In addition, participants were asked to complete a daily log book where they recorded their waking hours. On workdays, participants recorded the times they started and finished work, along with the times of their breaks during the day. Information about any non-wear time was also recorded in the daily $\log$. At the end of the seven days the devices and diary were collected from the participants.

\section{Data processing}

Downloaded data from the activPAL were processed manually using a customized Microsoft Excel macro. The activPAL data which are downloaded into 15-sec epochs were re-integrated and summarized (using the Microsoft Excel macro) over 60-sec epochs (to match the ActiGraph data) and time spent sitting, standing and stepping, including number of steps and average cadence and sit-to-stand transitions were extracted. Sleeping time was identified as the last transition from standing to sitting/lying and the first transition from sitting/lying to standing during the time that best matched the participants' daily log. For each identified sleeping bout, data were explored 60 minutes before and after and included as sleeping time if sitting/lying time was $\geq 30$ minutes and $<20$ steps were recorded. If any standing time with $<20$ steps was found during sleeping hours, this was considered as sleeping time. Non-wear time was considered as time spent in either a sitting/lying or standing position for $\geq 3$ hours, with no transitions. 

determined by summing the minutes spent below the 6-different cut-points defined above. Sleep time was interpreted as the consecutive strings of sedentary minutes during the night time that best matched the activPAL data. Sleep time was excluded from the analysis. Nonwear time data (continuous strings of zero counts) that best matched the activPAL data was removed from the analysis.

To be included in the analyses participants were required to have worn both devices concurrently for at least 600 minutes on at least 4 days, including three work days and one non-work day. For each participant, time matched total minutes spent sedentary during working hours and outside of working hours on workdays, and total sedentary and nonsedentary time on non-workdays were extracted based on times derived from participant's logs. Timed-matched data for waking hours during work and non-workdays and during working and non-working hours were retrieved from the activPAL and ActiGraph. These were analysed for each participant using a Microsoft Excel macro which summarised total activPAL and ActiGraph-determined sedentary time for each domain.

\section{Data analysis}

Sedentary times determined by the activPAL and ActiGraph during waking hours on workdays and non-workdays, and during working-hours and non-working-hours on workdays were analysed using SPSS version 22. These variables were tested for normality using the Shapiro-Wilk Test, which confirmed that all data were normally distributed. between instruments on workdays and non-workdays and during working hours and non- 
working hours on workdays. The Cohen's $d$ statistic has been reported to provide a further insight into the magnitude of the differences between the activPAL and ActiGraph, by providing information on the standardised difference between the means. An effect size calculator (UCCS Lee Becker) was used to determine the effect sizes. A small effect is classified as 0.2 , a medium effect as 0.5 and a large effect is classified as 0.8 (Cohen, 1988).

The coefficient of variation was used to assess the variability of the standard deviation with respect to the mean.

The mean difference in time spent sedentary within the different domains, along with the 95\% limits of agreement, were calculated using Bland-Altman plots (Bland and Altman, 1986). In addition, to assess the agreement of total sedentary time between the outputs of the two devices, two-way mixed Intraclass Correlation Coefficients (ICC) were calculated. ICC results were interpreted as follows: 0-0.39 indicates poor agreement: 0.40.59 indicates fair agreement; 0.6-0.74 indicates moderate agreement; and $>0.75$ indicates excellent agreement (Cicchetti, 1994). Receiver operating characteristic (ROC) curve analyses $(95 \% \mathrm{Cl})$ (Jago et al., 2007) were conducted to explore the sensitivity and specificity of each of the cut-points applied to the ActiGraph data to determine sedentary time. The area under the curve value (AUC; representing the percentage of time that the ActiGraph correctly identified sedentary time) was used to determine the accuracy of the diagnostic ability of the ActiGraph compared with the activPAL. These tests were used to examine if the ActiGraph cut-points correctly identify sedentary and non-sedentary time compared to the activPAL during workdays and non-workdays (Jago et al., 2007). Statistical significance was set at $p<0.05$. Results are presented as mean (SD) unless stated otherwise. The ActiGraph and activPAL outputs were plotted against each other as a Figure for one person 
during a workday and a non-workday to illustrate the level of agreement between both devices.

\section{Results}

Of the 35 drivers enrolled in the study, 28 (100\% male; mean age 43.9 (27) years; mean BMI $28.5(3.9) \mathrm{kg} / \mathrm{m}^{2}$ ) provided at least 4 days of valid data for both monitors. The average wear times during waking hours for the activPAL and ActiGraph for workdays were 1014(57) minutes/day (working-hours: 604(85) minutes/day) and 869(295) minutes/day for non-workdays. ActiGraph cut-points during each of the different domains over the monitoring period are shown in Table 1. Bland-Altman plots depicting the mean differences and $95 \%$ limits of agreement between sedentary times measured using the different ActiGraph cut-points and the activPAL on workdays and non-workdays, and during working hours and non-working hours on workdays are shown in Figures 1 and 2. sedentary time during workdays when using the $<50 \mathrm{cpm},<100 \mathrm{cpm}$ and the $<150 \mathrm{cpm}$ cutpoints. All ActiGraph cut-points significantly underestimated sedentary time during working hours (Table 1). Outside of working hours on workdays, and during non-workdays, no 
significant differences were observed between sedentary times estimated using the $<50$ cpm cut-point in comparison to the activPAL. All other ActiGraph cut-points significantly overestimated sedentary time, relative to the activPAL on non-workdays, and during nonworking hours on workdays. Effects sizes (Cohen's d) for the differences in sedentary times between the devices ranged from small (0.1) to large (0.8), depending on the cut-point

(Table 1). The strength of the associations, as determined by the ICC, between ActiGraphdetermined sedentary time and sitting time measured using the activPAL were stronger on non-workdays, and during non-working hours on workdays, across all cut-points in comparison to workdays and working hours on workdays (Table 1).

Sensitivity and specificity of ActiGraph-determined sedentary time

Figure 3 shows the area under the ROC curve for the ActiGraph during workdays, which showed poor discrimination of sedentary time compared with the activPAL (ROC= .617). Despite the different cut-points generally showing better sensitivity than specificity, the sensitivity and specificity values across all cut-points were low (Table 2). Figure 4 shows the area under the ROC curve for the ActiGraph during non-workdays, which showed better discrimination of sedentary time compared to workdays $(\mathrm{ROC}=.706$ versus $\mathrm{ROC}=.617)$. As with the workday data, the sensitivity and specificity values across all cut-points were low (Table 3).

Figure 5 shows the ActiGraph output illustrating activity patterns on a typical

\section{Illustration of activity patterns on a typical workday and non-workday for a bus driver} workday and non-workday for a bus driver compared with the activPAL. Sedentary time 
identified by the activPAL is represented by a straight line. ActiGraph and activPAL outputs are superposed and time-matched for waking hours during a typical workday and nonworkday.

\section{Discussion}

The aim of this study was to explore the validity of accelerometer-determined freeliving sedentary time (assessed using $<50 \mathrm{cpm},<100 \mathrm{cpm},<150 \mathrm{cpm},<200 \mathrm{cpm},<250 \mathrm{cpm}$ and $<300 \mathrm{cpm}$ cut-points) during and outside working hours in comparison to the activPAL in a sample of bus drivers. All ActiGraph cut-points assessed in this study significantly underestimated sedentary time during working hours, which led to weak associations between accelerometer-determined sedentary time and activPAL determined sitting time on workdays. Whilst closer agreements were observed between accelerometer-determined sedentary time and the activPAL on non-workdays, ROC analyses revealed that the ActiGraph generally showed poor discrimination of sedentary time compared with the activPAL on both workdays and non-workdays. These findings suggest that the ActiGraph is not a valid tool to assess sedentary time in bus drivers, particularly during workdays.

Kozey-Keadle and colleagues identified in 2011 significant differences in ActiGraphdetermined sedentary time depending on which cut-point was applied to the data (ranging from $<50 \mathrm{cpm}$ to $<250 \mathrm{cpm}$ ) over a 6-hour direct observation period. In their study, which involved simulated office work, the authors reported that the cut-point with the lowest bias was the $<150 \mathrm{cpm}$ cut-point, which overestimated sedentary time by $1.8 \%$. In contrast, the most commonly used $<100 \mathrm{cpm}$ cut-point underestimated sedentary time by $4.9 \%$. In a different study which compared sedentary time estimated by different ActiGraph cut-points 
to activPAL-determined sitting time in free-living adults, Hart et al. (2011). observed an ActiGraph cut-point of $<50 \mathrm{cpm}$ had the closest agreement with the activPAL.

In contrast to the above studies, the present paper examined the validity of different

ActiGraph cut-points in a different occupational group comprising bus drivers. In the present study, discrepancies in the most accurate ActiGraph cut-point for detecting sedentary time, relative to the activPAL, existed between workdays and non-workdays. On workdays, the $<250 \mathrm{cpm}$ cut-point provided the closest estimate of total daily sedentary time compared to the activPAL (mean difference +17 minutes/day). However, on non-workdays total daily sedentary time was best estimated using the $<50 \mathrm{cpm}$ cut-point, which underestimated sedentary time by 34 minutes/day. During working hours, all cut-points underestimated sedentary time relative to the activPAL, with the $<300 \mathrm{cpm}$ cut-point providing the closest estimate (underestimating by 33 mins/day).

Wong et al. (2014) used the ActiGraph-accelerometer $(<150 \mathrm{cpm})$ to measure sedentary time in a sample of bus drivers in Australia. In this study, they showed that, despite the sedentary nature of a driving occupation, bus drivers accumulated less sedentary time during workdays in comparison to non-workdays (7.8 hours/day versus 8.9hours/day, respectively) and during working hours compared to non-working hours (3.7 hours/day versus 4.1 hours/day, respectively). These results are contrary to those presented in this study, which revealed that drivers spent 715 minutes ( 12 hours/day) sitting on workdays and 536 minutes ( 8.8 hours/day) sitting on non-workdays, and 478 minutes (7.9 hours/day) sitting during working hours. Taking into account any potential cultural or weather-related (UK and Australia) differences between these studies; these results suggest that using accelerometer-applied cut-points to determine sedentary time (as done by Wong 
and colleagues, 2014) may result in the underestimation of sedentary time, possibly due to the misclassification of sedentary time as light physical activity in this occupational group, due to the movement of the bus.

The ActiGraph accelerometer records the intensity of the body's movements and sedentary time is estimated through a lack of movement counts (Chen et al., 2005). Any motion over any of the cut-points applied within this sample of drivers will therefore be considered as non-sedentary activity and clustered as light-activity, as indicated by the findings of the current paper. Discrepancies between the activPAL and the ActiGraph could therefore be due to the vibrations experienced during vehicle motion. Figure 5 illustrates the ActiGraph output activity patterns on a typical workday and non-workday for a bus driver, compared to the activPAL. This figure indicates that continuous accelerations during working-hours are detected by the ActiGraph and classified as non-sedentary time, whilst the time-matched activPAL output clustered this time as sitting based on the body's posture. Therefore, it is plausible that the discrepancies detected between the activPAL and threshold, despite the driver being seated (Patterson et al., 1993).

This study provides novel information on sedentary behaviour measurement and how sedentary time is accumulated during and outside working hours using two different tools in a sample of bus drivers. This study is not without limitations however. One limitation is that the bus vibrations were not objectively quantified and it is difficult therefore to assess their impact on the monitor's sensitivity to accurately detecting 
sedentary time. Moreover, the sample size of this study was relatively small and included participants who were overweight or obese, which may have exacerbated the impact of the

339 vehicle vibrations on the monitor's performance. This issue should be investigated further in 340 larger samples and in other driving occupations.

\section{Conclusions}

The present paper highlights that the ActiGraph accelerometer, relative to the 344 activPAL, misclassifies sedentary time in bus drivers, particularly during working hours.

345 When the use of the activPAL is not possible in such occupational groups, further research

346 should explore a correction formula to apply to ActiGraph data for defining sedentary time

347 in occupational drivers. Overall, sedentary behaviour measurement techniques should be 348 improved in this understudied, highly sedentary and at risk occupational group. 
Atkin, A. J., Gorely, T., Clemes, S. A., Yates, T., Edwardson, C., Brage, S., et al. (2012). Methods of measurement in epidemiology: Sedentary behaviour. International Journal of Epidemiology, 41(5), 1460-1471.

Berendsen, B.A., Hendriks, M.R., Meijer, K., Plasqui, G., Schaper, N.C. and Savelberg, H.H., 2014. Which activity monitor to use? Validity, reproducibility and user friendliness of three activity monitors. BMC Public Health, 14(1), p.749.

Biswas, A., Oh, P. I., Faulkner, G. E., Bajaj, R. R., Silver, M. A., Mitchell, M. S., et al. (2015). Sedentary time and its association with risk for disease incidence, mortality, and hospitalization in adults: A systematic review and meta-analysis. Annals of Internal Medicine, 162(2), 123-132.

Bland, J. M., \& Altman, D. G. (1986). Statistical methods for assessing agreement between two methods of clinical measurement. Lancet (London, England), 1(8476), 307-310. Brocklebank, L. A., Falconer, C. L., Page, A. S., Perry, R., \& Cooper, A. R. (2015). Accelerometer-measured sedentary time and cardiometabolic biomarkers: A systematic review. Preventive Medicine, 76, 92-102.

372 Brown, H. E., Ryde, G. C., Gilson, N. D., Burton, N. W., \& Brown, W. J. (2013). Objectively measured sedentary behavior and physical activity in office employees: Relationships with 374 presenteeism. Journal of Occupational and Environmental Medicine / American College of Occupational and Environmental Medicine, 55(8), 945-953.

Chen, K. Y., \& Bassett, D. R.,Jr. (2005). The technology of accelerometry-based activity monitors: Current and future. Medicine and Science in Sports and Exercise, 37(11 Suppl), S490-500. 
Q., et al. (2011). Trends over 5 decades in U.S. occupation-related physical activity and their associations with obesity. PloS One, 6(5), e19657.

Clemes, S. A., Houdmont, J., Munir, F., Wilson, K., Kerr, R., \& Addley, K. (2015). Descriptive epidemiology of domain-specific sitting in working adults: The stormont study. Journal of Public Health (Oxford, England).

Cicchetti, D.V., (1994). Guidelines, criteria, and rules of thumb for evaluating normed and standardized assessment instruments in psychology. Psychological assessmen, 6(4), 284.

Cohen, J. (1988). Statistical power analysis for the behavioral sciences (2nd ed.). Hillsdale, NJ: Lawrence Earlbaum Associates.

De Decker, E., De Craemer, M., Santos-Lozano, A., Van Cauwenberghe, E., De Bourdeaudhuij, I. and Cardon, G. (2013). Validity of the ActivPAL ${ }^{\mathrm{TM}}$ and the ActiGraph monitors in preschoolers. Medicine and science in sports and exercise45(10), 2002-2011.

Dunstan, D. W., Wiesner, G., Eakin, E. G., Neuhaus, M., Owen, N., LaMontagne, A. D., et al. (2013). Reducing office workers' sitting time: Rationale and study design for the stand up victoria cluster randomized trial. BMC Public Health, 13, 1057-2458-13-1057.

French, S. A., Harnack, L. J., Toomey, T. L., \& Hannan, P. J. (2007). Association between body weight, physical activity and food choices among metropolitan transit workers. The International Journal of Behavioral Nutrition and Physical Activity, 4, 52.

Godfrey, A., Culhane, K. M., \& Lyons, G. M. (2007). Comparison of the performance of the activPAL professional physical activity logger to a discrete accelerometer-based activity monitor. Medical Engineering \& Physics, 29(8), 930-934. 

activity monitor in the measurement of posture and motion during everyday activities. British Journal of Sports Medicine, 40(12), 992-997.

Hart, T. L., McClain, J. J., \& Tudor-Locke, C. (2011). Controlled and free-living evaluation of objective measures of sedentary and active behaviors. Journal of Physical Activity \& Health, 8(6), 848-857.

407

Healy, G. N., Matthews, C. E., Dunstan, D. W., Winkler, E. A., \& Owen, N. (2011). Sedentary time and cardio-metabolic biomarkers in US adults: NHANES 2003-06. European Heart Journal, 32(5), 590-597.

Jago, R., Zakeri, I., Baranowski, T. and Watson, K., (2007). Decision boundaries and receiver operating characteristic curves: new methods for determining accelerometer cutpoints. Journal of sports sciences, 25(8), 937-944. John, L.M., Flin, R. and Mearns, K., (2006). Bus driver well-being review: 50 years of research. Transportation Research Part F: Traffic Psychology and Behaviour, 9(2), 89-114. Joshi, B.A., Joshi, A.V., Katti, S.M., Mallapur, M.D. and Karikatti, S.S., (2013). A crosssectional study of prevalence of overweight and obesity among bus drivers and conductors of North-West Karnataka Road Transport Corporation (NWKRTC) in Belgaum Division, Belgaum. Journal of the Indian Medical Association, 111(3), 157-159. from all causes, cardiovascular disease, and cancer. Medicine and Science in Sports and Exercise, 41(5), 998-1005.

Kozey, S. L., Lyden, K., Howe, C. A., Staudenmayer, J. W., \& Freedson, P. S. (2010). Accelerometer output and MET values of common physical activities. Medicine and Science in Sports and Exercise, 42(9), 1776-1784. 

(2008). Amount of time spent in sedentary behaviors in the United States, 2003-2004. American journal of epidemiology, 167(7), 875-881. guidelines of the european society of hypertension for clinic, ambulatory and self blood pressure measurement. Journal of Hypertension, 23(4), 697-701.

Oliver, M., Schofield, G. M., Badland, H. M., \& Shepherd, J. (2010). Utility of accelerometer thresholds for classifying sitting in office workers. Preventive Medicine, 51(5), 357-360.

Patterson, S.M., Krantz, D.S., Montgomery, L.C., Deuster, P.A., Hedges, S.M. and Nebel, L.E., (1993). Automated physical activity monitoring: Validation and comparison with physiological and self-report measures. Psychophysiology, 30(3), 296-305.

Ridgers, N. D., Salmon, J., Ridley, K., O'Connell, E., Arundell, L., \& Timperio, A. (2012). Agreement between activPAL and ActiGraph for assessing children's sedentary time. The International Journal of Behavioral Nutrition and Physical Activity, 9, 15-5868-9-15. Sedentary Behaviour Research, N. (2012). Letter to the editor: Standardized use of the 440 terms "sedentary" and "sedentary behaviours". Applied Physiology, Nutrition, and Metabolism = Physiologie Appliquee, Nutrition Et Metabolisme, 37(3), 540-542.

Thorp, A. A., Healy, G. N., Winkler, E., Clark, B. K., Gardiner, P. A., Owen, N., et al. (2012). Prolonged sedentary time and physical activity in workplace and non-work contexts: A cross-sectional study of office, customer service and call centre employees. The International Journal of Behavioral Nutrition and Physical Activity, 9, 128-5868-9-128. means and standard deviations. [Date; cited 201721 March]. Available from: 
449 Varela-Mato, V., Yates, T., Stensel, D., Biddle, S., \& Clemes, S. (2015). Time spent sitting 450 during and outside working hours in bus drivers: A pilot study. Preventive Medicine 451 Reports, Volume 3, 36-39.

452 Wilmot, E. G., Edwardson, C. L., Achana, F. A., Davies, M. J., Gorely, T., Gray, L. J., et al. 453 (2012). Sedentary time in adults and the association with diabetes, cardiovascular disease 454 and death: Systematic review and meta-analysis. Diabetologia, 55(11), 2895-2905

455 Wong, J. Y., Gilson, N. D., Bush, R. A., \& Brown, W. J. (2014). Patterns and perceptions of 456 physical activity and sedentary time in male transport drivers working in regional 457 australia. Australian and New Zealand Journal of Public Health, 38(4), 314-32. 
Table 1. Mean (SD) time spent sedentary in each domain recorded by the activPAL and ActiGraph. ICCs, t-test (95\% Cl) and Cohen's d results are based on the comparisons between the times spent sedentary (mins/day) measured using the different ActiGraph cut-points and sedentary time measured using the activPAL (the criterion)

\begin{tabular}{|c|c|c|c|c|c|c|c|c|}
\hline Domains & $\begin{array}{l}\text { ActiGraph } \\
\text { cut-points } \\
\text { (cpm) }\end{array}$ & $\begin{array}{l}\text { ActiGraph- } \\
\text { determined } \\
\text { sedentary time } \\
\text { (min/day) }\end{array}$ & $\begin{array}{c}\text { activPAL- } \\
\text { determined } \\
\text { sedentary time } \\
\text { (min/day) }\end{array}$ & $\begin{array}{l}\text { ICC between } \\
\text { devices }\end{array}$ & $\begin{array}{l}\mathrm{CV} \\
(\%)\end{array}$ & $\begin{array}{l}\mathrm{p} \text { value } \\
\text { (t-test) }\end{array}$ & $\begin{array}{l}95 \% \mathrm{Cl} \\
\text { (t-test) }\end{array}$ & $\begin{array}{l}\text { Cohen's d } \\
\text { (effect } \\
\text { size) }\end{array}$ \\
\hline \multirow{6}{*}{$\begin{array}{l}\text { Total waking hours } \\
\text { on workdays }\end{array}$} & $<50$ & $480(92)$ & & .261 & 14.2 & $<.001$ & $-287.0,-182.5$ & 0.7 \\
\hline & $<100$ & $572(96)$ & & .334 & 14.8 & $<.001$ & $-193.1,-92.4$ & 0.5 \\
\hline & $<150$ & $638(95)$ & $715(123)$ & .400 & 14.7 & $<.010$ & $-124.6,-29.4$ & 0.3 \\
\hline & $<200$ & $690(91)$ & & .450 & 14.1 & .274 & $-69.5,20.6$ & 0.1 \\
\hline & $<250$ & $732(84)$ & & .481 & 13.0 & .424 & $-26.1,60.2$ & -0.1 \\
\hline & $<300$ & $768(84)$ & & .523 & 13.0 & .012 & $12.5,94.1$ & -0.2 \\
\hline \multirow{6}{*}{$\begin{array}{l}\text { Total waking hours } \\
\text { on non-workdays }\end{array}$} & $<50$ & 502(169) & & .775 & 26.1 & .170 & $-83.7,15.6$ & 0.1 \\
\hline & $<100$ & 594(199) & & .879 & 32.4 & $<.010$ & $18.2,96.5$ & -0.1 \\
\hline & $<150$ & $619(206)$ & $536(203)$ & .887 & 33.6 & $<.001$ & $44.1,121.2$ & -0.2 \\
\hline & $<200$ & $641(212)$ & & .888 & 34.6 & $<.001$ & $65.5,143.1$ & -0.2 \\
\hline & $<250$ & $662(216)$ & & .910 & 35.2 & $<.001$ & $90.3,160.6$ & -0.3 \\
\hline & $<300$ & $663(217)$ & & .911 & 35.4 & $<.001$ & $91.1,161.4$ & -0.3 \\
\hline \multirow{6}{*}{$\begin{array}{l}\text { Working hours on } \\
\text { workdays }\end{array}$} & $<50$ & $229(52)$ & & .118 & 8.49 & $<.001$ & $-293.1,-205.3$ & 0.8 \\
\hline & $<100$ & $298(65)$ & & .300 & 18.3 & $<.001$ & $-221.6,-139.6$ & 0.7 \\
\hline & $<150$ & $348(68)$ & $478(106)$ & .418 & 19.2 & $<.001$ & $-168.6,-92.8$ & 0.6 \\
\hline & $<200$ & $388(69)$ & & .496 & 19.5 & $<.001$ & $-125.9,-54.9$ & 0.5 \\
\hline & $<250$ & $420(70)$ & & .552 & 19.7 & $<.010$ & $-92.4,-25.3$ & 0.3 \\
\hline & $<300$ & $445(71)$ & & .593 & 20.0 & .042 & $-65.5,-1.3$ & 0.2 \\
\hline Non-working hours & $<50$ & $251(75)$ & & .727 & 21.1 & .158 & $-6.1,34.9$ & -0.1 \\
\hline \multirow{5}{*}{ on workdays } & $<100$ & $274(81)$ & & .739 & 27.7 & $<.010$ & $16.8,58.9$ & -0.2 \\
\hline & $<150$ & $290(84)$ & $236(65)$ & .747 & 28.8 & $<.001$ & $32.7,74.8$ & -0.3 \\
\hline & $<200$ & $302(85)$ & & .752 & 29.1 & $<.001$ & $44.9,86.9$ & -0.4 \\
\hline & $<250$ & $312(86)$ & & .752 & 29.5 & $<.001$ & $54.7,97.1$ & -0.4 \\
\hline & $<300$ & $323(84)$ & & .764 & 28.8 & $<.001$ & $66.3,107.1$ & -0.5 \\
\hline
\end{tabular}

Abbreviations: ICC, intraclass correlation coefficient. Cl, confidence interval; CV, coefficient of variation 
Workdays

$50 \mathrm{cpm}$
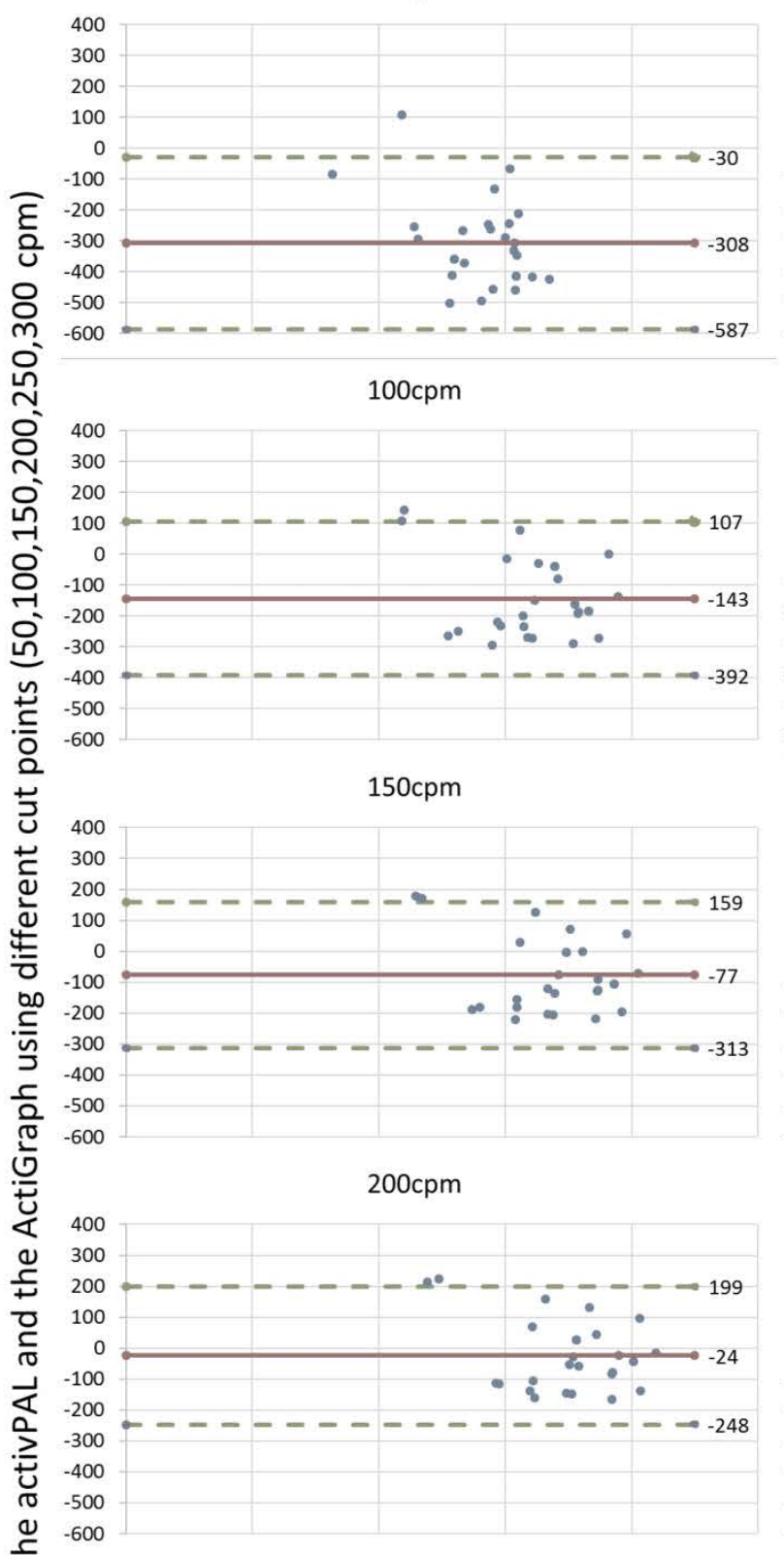

(

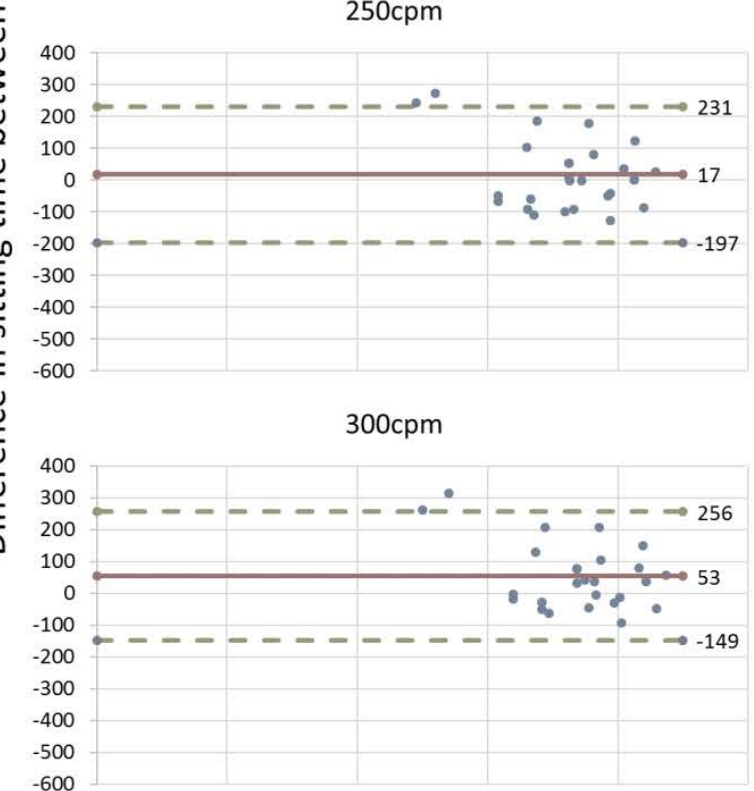

Non-Workdays

$50 \mathrm{cpm}$

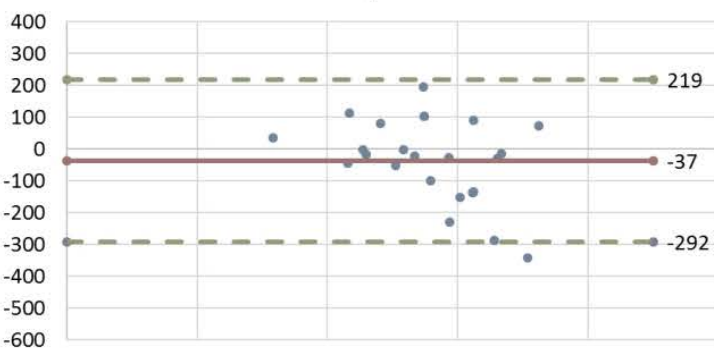

$100 \mathrm{cpm}$

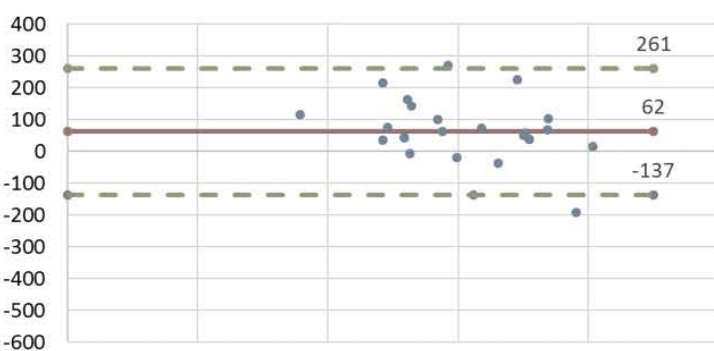

$150 \mathrm{cpm}$

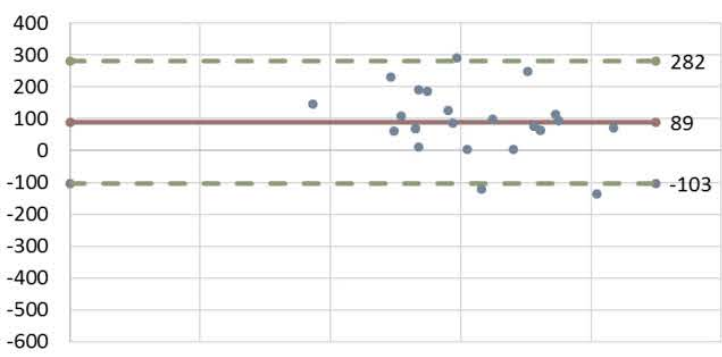

$200 \mathrm{cpm}$

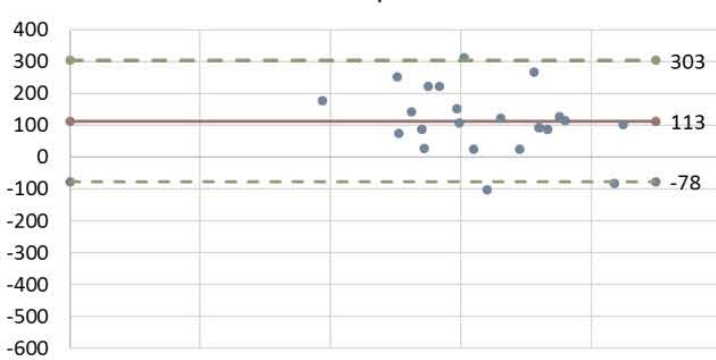

$250 \mathrm{cpm}$

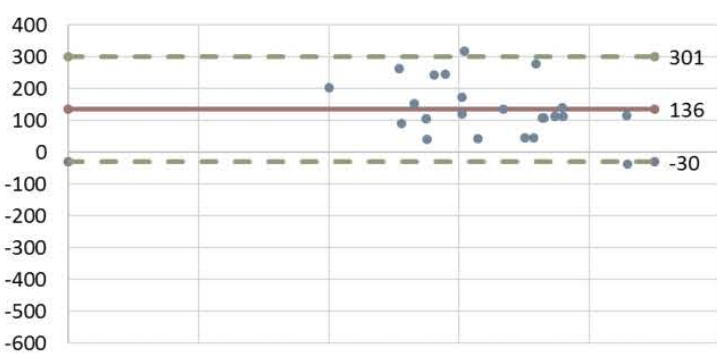

$300 \mathrm{cpm}$

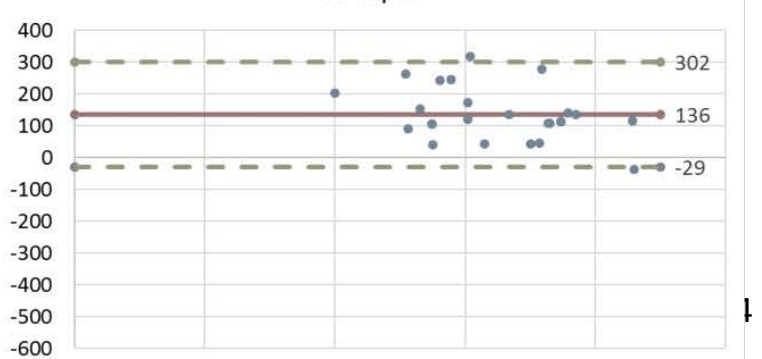


460

Figure 1. Bland-Altman plots showing the mean difference and the $95 \%$ limits of 461 agreement in time spent sedentary on workdays and non-workdays, between the ActGraph GT3X+ (using different cut points) and the activPAL. The straight lines

462 represent the mean difference in minutes between the activPAL and the ActiGraph and the dotted lines represent the $95 \%$ confidence intervals of the agreement between the

463 measures. The $x$ axis represents the mean sedentary time calculated between devices and the $y$ axis is the difference in sedentary time (in minutes) between the ActiGraph 464 and the activPAL.

465

466

467

468

469

470

471

472

473

474

475

476 
Working-hours

478
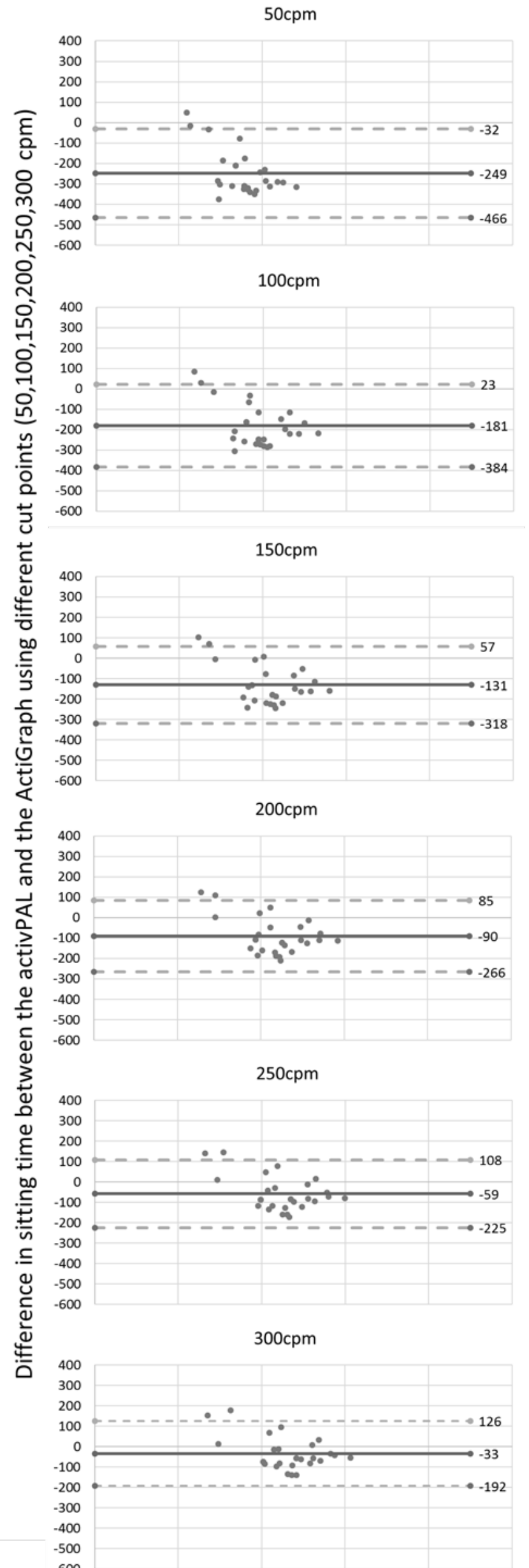

Non-Working-hours

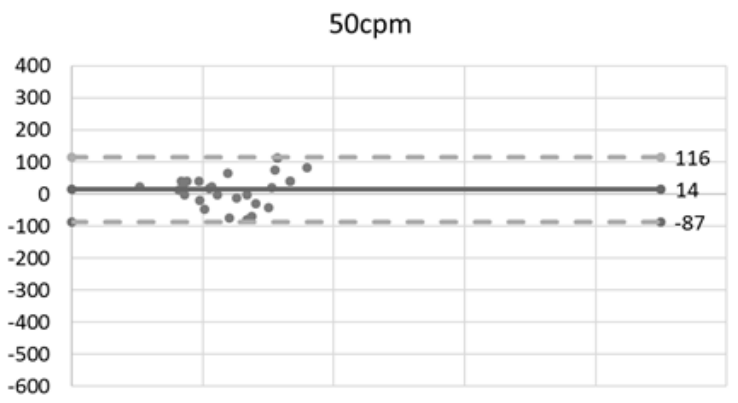

$100 \mathrm{cpm}$

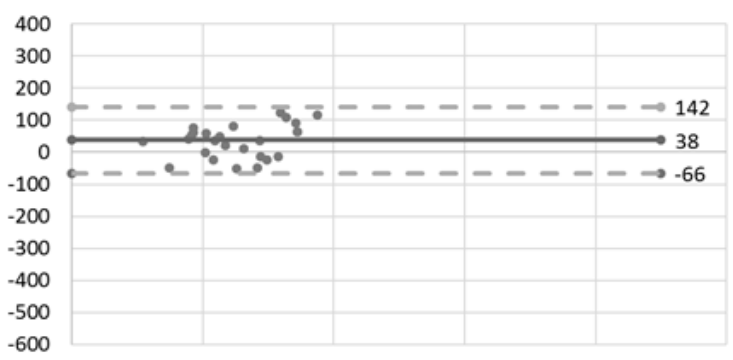

$150 \mathrm{cpm}$

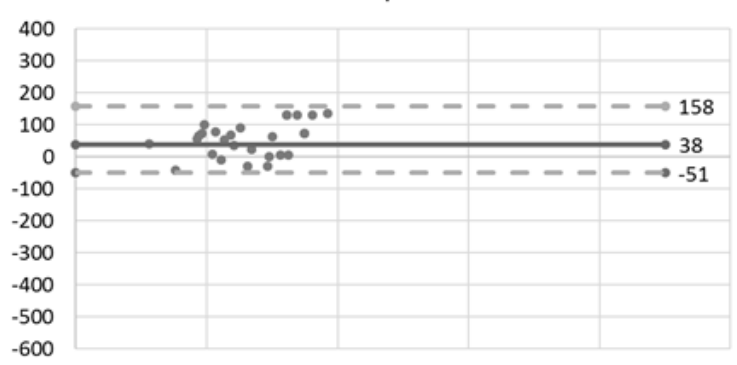

$200 \mathrm{cpm}$

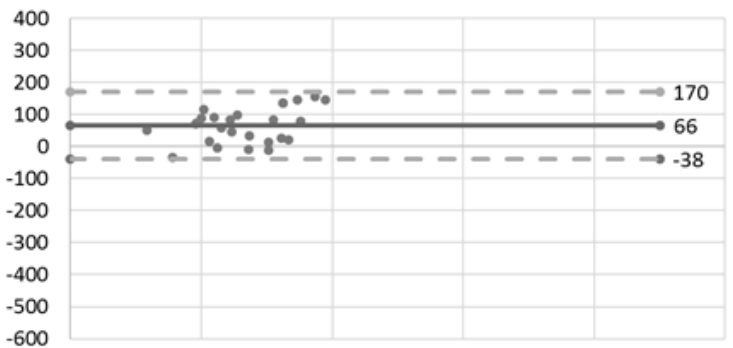

$250 \mathrm{cpm}$

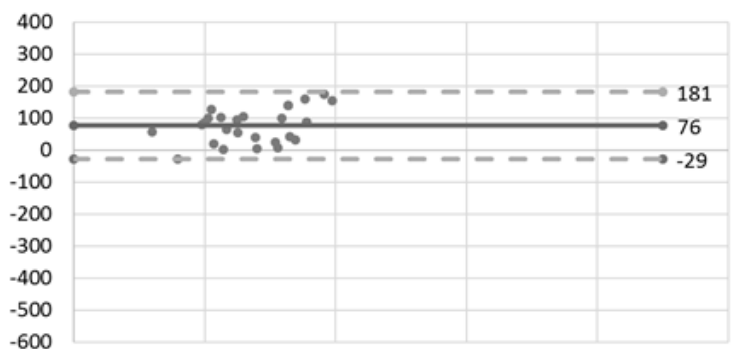

$300 \mathrm{cpm}$

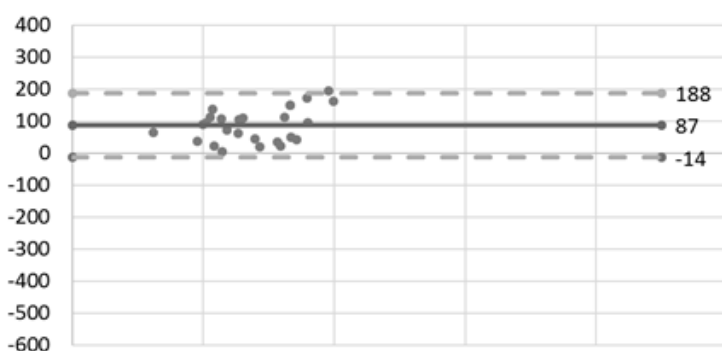


Figure 2. Bland-Altman plots showing the mean difference and the $95 \%$ limits of 480 agreement in time spent sedentary during working-hours and non-working-hours on workdays, between the ActGraph GT3X+ (using different cut-points) and the activPAL. The straight lines represent the mean difference in minutes between the activPAL and the ActiGraph and the dotted lines represent the 95\% confidence intervals of the agreement between the measures. The $x$ axis represents the mean sedentary time between devices and the $y$ axis is the difference in sedentary time (in minutes) between the ActiGraph and the activPAL.

484

485

486

487

488

489

490

491

492

493

494

495

496

497

498

499

500

501

502

503

504

505

506

507

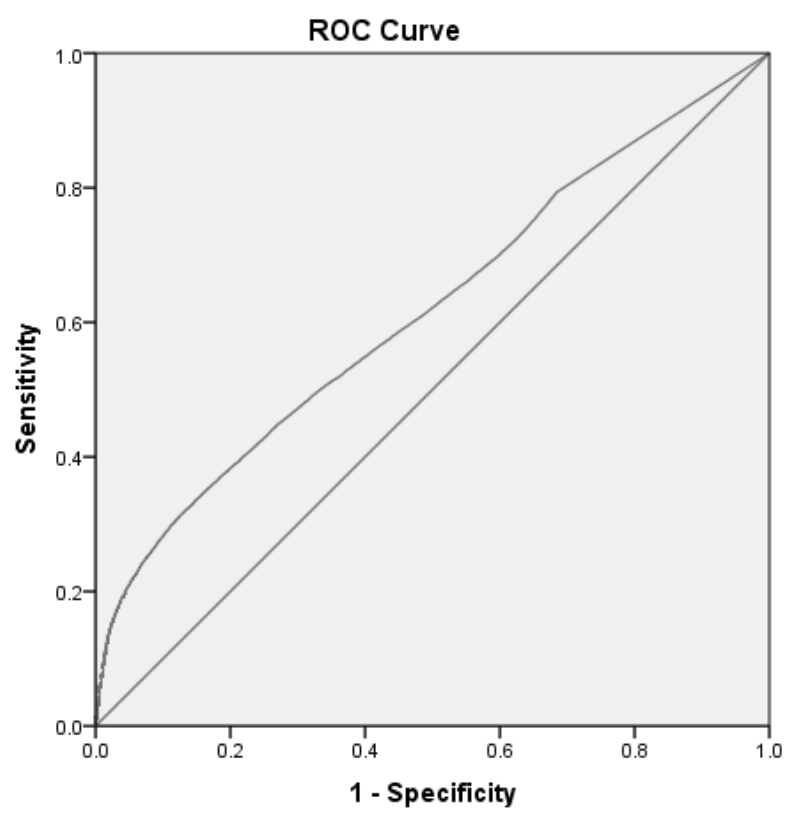

Figure 3. ROC curve for ActiGraph-determined sedentary time during workdays 
Table 2. Sensitivity and specificity values calculated across participants for each ActiGraph cut-point. The performance of each cut-point was compared to activPALdetermined total daily sitting time on workdays. The area under the ROC curve value to assess the accuracy of the ActiGraph to detect sitting time compared with the activPAL.

\begin{tabular}{lcc} 
Area under the ROC curve $(95 \% \mathrm{Cl})$ & \multicolumn{2}{c}{$.617(.613-.621)$} \\
\hline $\begin{array}{l}\text { ActiGraph-determined } \\
\text { sedentary cut points }\end{array}$ & Sensitivity & Specificity \\
\hline$<50 \mathrm{cpm}$ & .620 & .498 \\
$<100 \mathrm{cpm}$ & .549 & .400 \\
$<150 \mathrm{cpm}$ & .498 & .331 \\
$<200 \mathrm{cpm}$ & .453 & .277 \\
$<250 \mathrm{cpm}$ & .413 & .234 \\
$<300 \mathrm{cpm}$ & .380 & .197
\end{tabular}

508

509

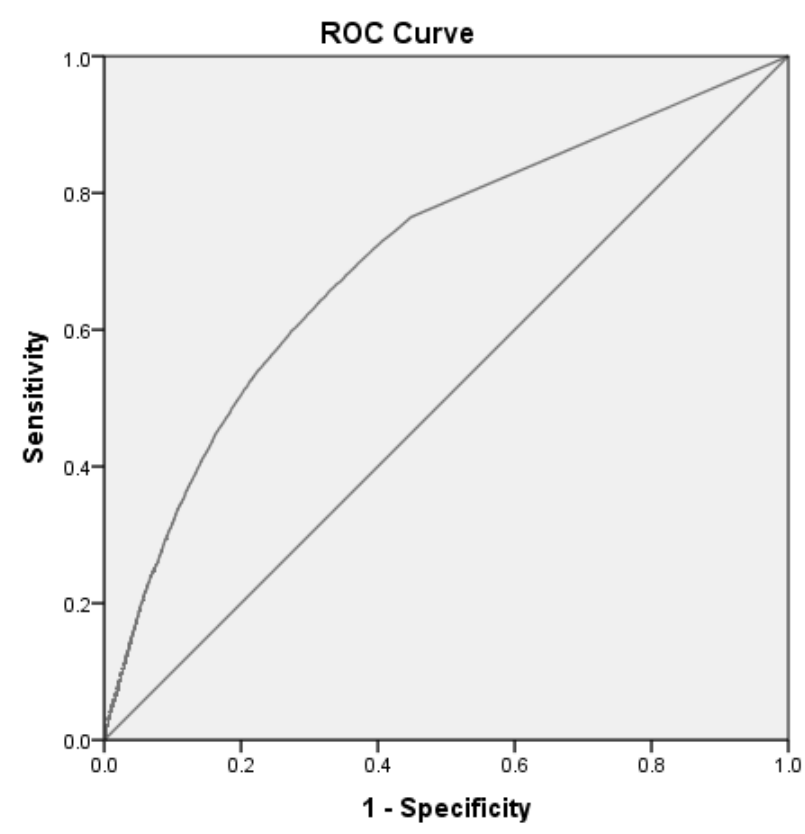

Figure 4. ROC curve for ActiGraph-determined sedentary time during non-workdays 
Table 3. Sensitivity and specificity values calculated across participants for each ActiGraph cut-point. The performance of each cut-point was compared to activPALdetermined total daily sitting time on non-workdays. The area under the ROC curve value to assess the accuracy of the ActiGraph to detect sitting time compared with the activPAL.

\begin{tabular}{lcc}
\hline \multicolumn{1}{c}{ Area under the ROC curve $(95 \% \mathrm{Cl})$} & $.706(.701-.712)$ \\
\hline $\begin{array}{l}\text { ActiGraph-determined sedentary cut } \\
\text { points }\end{array}$ & Sensitivity & Specificity \\
\hline$<50$ cpm & .602 & .278 \\
$<100 \mathrm{cpm}$ & .532 & .219 \\
$<150 \mathrm{cpm}$ & .478 & .182 \\
$<200 \mathrm{cpm}$ & .437 & .158 \\
$<250 \mathrm{cpm}$ & .401 & .139 \\
$<300 \mathrm{cpm}$ & .372 & .124 \\
\hline
\end{tabular}

521

522

523

524

525

526

527

528

529 
ActiGraph activity data compared against the activPAL during a workday
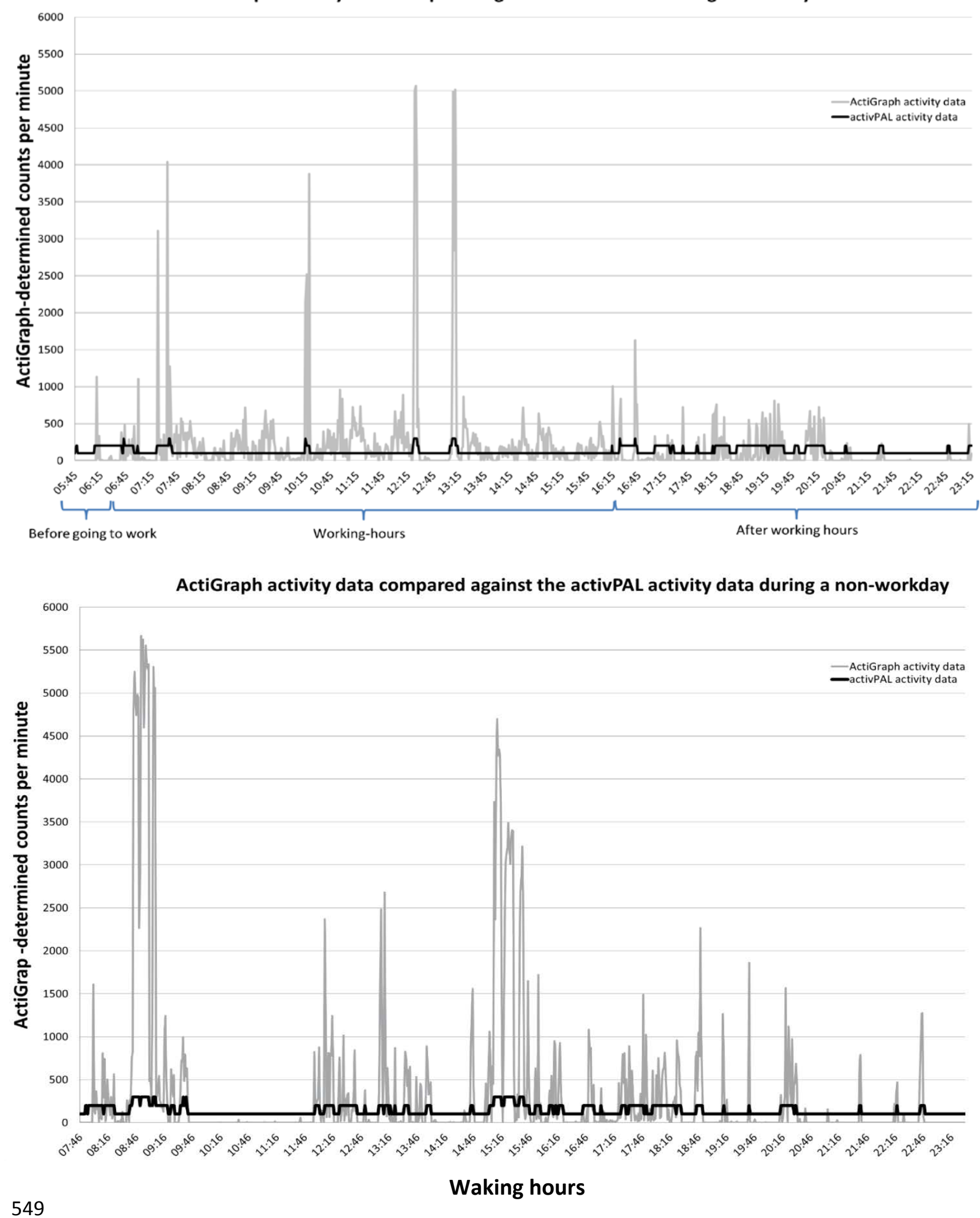

550 Figure 5. ActiGraph output illustrating activity patterns on a typical workday and non-

551 workday for a bus driver, compared to the activPAL. 